\title{
Caracterización en línea de la dinámica temporal de señales para el monitoreo del sistema eléctrico de potencia
}

\section{On-line characterization of temporal dynamic of signals for monitoring the electrical power system}

\author{
LEZAMA-ZÁRRAGA, Francisco Román†ं, CHAN-GONZÁLEZ, Jorge de Jesús, SHIH, Meng Yen y \\ CANTO-CANUL, Roberto Carlos
}

\author{
Universidad Autónoma de Campeche, Campus V, Predio s/n por Av. Humberto Lanz Cárdenas y Unidad Habitacional \\ Ecológica Ambiental, Col. Ex-Hacienda Kala, CP. 24085, San Francisco de Campeche, Cam., México.
}

ID $1^{\text {er }}$ Autor: Francisco Román, Lezama-Zárraga / ORC ID: 0000-0003-3397-7881, Researcher ID Thomson: U-12292018, CVU CONACYT ID: 205493

ID $1^{\text {er }}$ Coautor: Jorge de Jesús, Chan-González / ORC ID: 0000-0002-8638-1646, CVU CONACYT ID: 84196

ID $2^{\text {do }}$ Coautor: Meng Yen, Shih / ORC ID: 0000-0001-7475-6458, CVU CONACYT ID: 408617

ID $3^{\text {er }}$ Coautor: Roberto Carlos, Canto-Canul / ORC ID: 0000-0003-2420-043X, CVU CONACYT ID: 391401

DOI: $10.35429 / J C P E .2020 .22 .7 .26 .34$

Recibido 25 de Enero, 2020; Aceptado 30 de Junio, 2020

\section{Resumen}

En el monitoreo de un sistema eléctrico de potencia, la caracterización de los fenómenos dinámicos es fundamental cuando está sujeto a disturbios. En este artículo se propone un enfoque sistemático para analizar y caracterizar en línea (On-line) la evolución temporal de oscilaciones no lineales y transitorios en dichos sistemas. Se emplean dos métodos; el primer método está basado en una descomposición local de la señal bajo estudio en funciones ortogonales base para la obtención de las dinámicas de oscilaciones transitorias. Seguidamente se aplica un segundo método a las funciones ortogonales base para obtener señales analíticas y caracterizar los atributos instantáneos amplitud, fase y frecuencia de las oscilaciones y determinar una interpretación física del comportamiento del sistema. La metodología propuesta es un análisis tiempo-frecuencia-energía el cual es posible aplicarlo en mediciones obtenidas de Unidades de Medición Fasorial sincronizados en tiempo. Los resultados muestran que la metodología propuesta proporciona una caracterización precisa de los fenómenos transitorios y con efectos no estacionarios.

\begin{abstract}
The characterization of dynamic phenomena is essential for monitoring the Electrical Power System subject to disturbances. This article proposes an On-line time systematic approach to analyze and characterize the temporal evolution of transient and nonlinear oscillations in these systems. Two methods are used; the first method is based on a local decomposition of the signal under study into orthogonal basis functions to obtain the dynamics of transient oscillations. Next, a second method is applied to those orthogonal basis functions to obtain analytical signals and characterize the instantaneous amplitude, phase and frequency attributes of the oscillations and determine a physical interpretation of the system's behavior. The proposed methodology is a time-frequencyenergy analysis which can be applied to the timesynchronized Phasor Measurement Units measurements. The results demonstrate that the proposed methodology provide an accurate characterization of transient phenomena with non-stationary effects.
\end{abstract}




\section{Introducción}

El estudio de las oscilaciones no lineales y no estacionarias en los sistemas eléctricos de potencia se ha convertido en objeto de intenso interés e investigación para los estudios de estabilidad de un sistema de potencia. En la práctica, la detección y caracterización de las oscilaciones no lineales e invariantes en el tiempo en los datos medidos es muy complicado por las variaciones no estacionarias en el comportamiento dinámico del sistema y requiere una capacidad computacional en tiempo real.

Diferentes técnicas de análisis no lineal y no estacionaria se han explorado en los últimos años como métodos prometedores para el análisis y monitoreo de sistemas eléctricos de potencia de área amplia (wide-area) (Hauer \& De Steesse, 2004), (Pierre, et al, 2007). En particular, la transformada de Hilbert-Huang (Hilbert-Huang transform, HHT) ha sido aplicada con gran éxito para datos medidos (Messina, 2009).

En este artículo se presenta un método eficaz para el análisis en línea de la dinámica local de oscilaciones transitorias utilizando una descomposición modal empírica (EMD) local y la transformada de Hilbert (HT). Mediante la combinación de una ventana deslizante de longitud finita que barre a toda la señal medida, se propone una aplicación local de la EMD y la transformada de Hilbert es usada a través de un filtro digital de respuesta-impulso-finita (FIR).

A diferencia de otros algoritmos de la transformada de Hilbert-Huang (Messina, et al 2006), este enfoque permite a la transformada de Hilbert-Huang ser una técnica de análisis en línea para datos medidos.

Para evaluar la precisión del enfoque propuesto, los resultados numéricos incluyen la estimación de la amplitud, la fase y frecuencia instantáneas de las oscilaciones obtenidas de unidades de medición fasorial (Phasor Measurement Units, PMUs) (Phadke, et al, 1983) ubicadas en el Sistema Eléctrico Nacional.

\section{Planteamiento del problema}

Se requiere una metodología para aislar y eliminar perturbaciones no lineales y no estacionarias, y transitorios en los sistemas eléctricos de potencia que las técnicas tradicionales no pueden eliminar durante su operación y monitoreo.

El algoritmo propuesto contiene poca carga computacional y puede ser implementado en línea para el monitoreo y control de los sistemas de medición de área amplia (Wide-Area Measurement Systems, WAMS). Los casos de estudio son eventos reales que han ocurrido en el Sistema Eléctrico Nacional (SEN) y se comparan los resultados con otras técnicas tradicionales.

\section{Análisis convencional de la transformada de Hilbert-Huang}

La transformada de Hilbert-Huang (HHT) es un método usado para el análisis de series de tiempo no lineales y no estacionarias, la cual está basada en la descomposición modal empírica (EMD) y la transformada de Hilbert (HT). La combinación de estas técnicas produce una caracterización dinámica de los principales patrones oscilatorios en las señales de los sistemas eléctricos de potencia. En esta sección, se proporciona una visión general de este método como un antecedente de su implementación en tiempo real.

La descomposición modal empírica (EMD) separa una señal en componentes de amplitud y frecuencia moduladas, que admiten en su interior un buen desempeño de la transformada Hilbert [11].

La EMD es una técnica iterativa y adaptiva, que descompone una señal real en un número de componentes de funciones intrínsecas modales (Intrinsecal Mode Functions, IMFs), con amplitudes y frecuencias variantes en el tiempo, a través de un proceso llamado sifting. La descomposición se puede representar matemáticamente como:

$x(t)=\sum_{j=1}^{n} c_{j}(t)+r_{n}(t)=\sum_{j=1}^{p} c_{j}(t)+\sum_{k=p+1}^{n} c_{k}(t)+r_{n}(t)$ 
Donde $n$ es el número de componentes modales, $c_{i}(t)$ son las IMFs con el comportamiento físico de interés, los términos restantes $c_{k}(t)$ son las IMFs no interesantes y $r_{n}(t)$ es el residuo.

El método EMD convencional implica esencialmente tres pasos:

- Dada la señal original $x(t)$, establecer $h_{i}(t)=x(t), \mathrm{y}$ extraer los mínimos $\mathrm{y}$ máximos locales de $h_{i}(t)$.

- Interpolar los mínimos y máximos locales con spline cúbicas para formar las envolventes superior e inferior, respectivamente.

- Obtener la media de las envolventes, $m_{i}(t)$ y restarlo de $h_{i}(t)$ para determinar una nueva función $h_{i+1}=h_{i}(t)-m_{i}(t)$.

El procedimiento de tres pasos se repite iterativamente hasta que cumpla los criterios de una IMF y luego $c_{j}(t)=h_{i+1}(t)$.

Una IMF debe cumplir con las siguientes condiciones:

Para toda la serie de tiempo el número de extremos y el número de cruce por cero difieren por, al menos, uno.

En cualquier punto de la serie temporal, el valor medio de la envolvente definida por los máximos locales y la envolvente definida por los mínimos locales es cero.

Una vez que la señal original $x(t)$ se descompone en un conjunto de IMFs, la transformada de Hilbert se aplica a cada componente para definir los atributos instantáneos.

La transformada de Hilbert (HT) de una señal se define como:

$\tilde{x}(t)=\frac{1}{\pi} P \int_{-\infty}^{\infty} \frac{x(\tau)}{\tau-t} d \tau=-\frac{1}{\pi} P \int_{-\infty}^{\infty} \frac{x(\tau)}{t-\tau} d \tau$

Esta ecuación, en la que se indica el valor principal de Cauchy (Huang, et al, 1998), es una integral impropia debido a una discontinuidad en el punto $t$, con $-\infty \leq t \leq+\infty$ por la singularidad en $\tau=t$.
En su forma discreta, la transformada de Hilbert se puede derivar en el dominio del tiempo, mediante la aplicación de una regla rectangular en la integral y así se obtiene como:

$$
\tilde{x}(t)=\widetilde{c_{J}} \approx \frac{2}{\pi} \sum_{k=0}^{\infty} \frac{1}{2 k+1}\left(x_{t+2 k+1}-x_{t-2 k-1}\right)
$$

La aplicación de la HT para el jth IMF produce una representación compleja o señal analítica $z_{j}(t)$, que se construye, colocando la HT $c_{j}(t)$ en la parte imaginaria de la señal analítica.

$$
\begin{gathered}
z_{j}(t)=c_{j}(t)+i \tilde{c}_{j}(t)=A_{j}(t) e^{i \phi_{j}(t)} \\
c_{j}(t)=A_{j}(t) \cos \left(\phi_{j}(t)\right) \\
\tilde{c}_{j}(t)=A_{j}(t) \sin \left(\phi_{j}(t)\right)
\end{gathered}
$$

Donde $A_{j}(t), \phi_{j}(t)$ y $\omega_{j}(t)$ son los atributos instantáneos amplitud o envolvente, la fase y la frecuencia del $c_{j}(t)$, respectivamente. A través de la ecuación (4) en su forma polar, es posible extraer los atributos instantáneos.

$$
\begin{gathered}
A_{j}(t)=\sqrt{c_{j}(t)^{2}+\tilde{c}_{j}(t)^{2}}, \\
\phi_{j}(t)=\tan ^{-1} \frac{\tilde{c}_{j}(t)}{c_{j}(t)}, \\
\omega_{j}(t)=\frac{d \phi_{j}(t)}{d t},
\end{gathered}
$$

Los cuales son calculados como funciones de tiempo y pueden por lo tanto ser relacionado para todos los eventos temporales durante la medición.

\section{Metodología propuesta: implementación en línea (On-line) de la HHT}

Tradicionalmente, el proceso sifting aplicado en la técnica EMD, se basa en la interpolación entre dos extremos (un máximo y un mínimo) sucesivos a lo largo de toda la señal. Esto puede dar lugar a una descomposición de la señal en pseudo-IMFs y otros efectos indeseables (Rilling, et al, 2003).

\section{EMD local}

Para evitar estos pseudo-IMFs se implementa una EMD local que subdivide la señal a través de un número finito de ventanas sobrepuestas de longitud constante. El tamaño de la ventana es $W_{k}=k \times \Delta t$ y la i-ésima ventana será $W_{i}=$ $\left[t_{i-k}, t_{i}\right]$. 
Esta ventana es deslizada a lo largo de la señal sobreponiéndola sobre las ventanas previas y luego la técnica de EMD se aplica a cada ventana para que sea una implementación local y no global. En la Figura 1 se muestra esta representación.

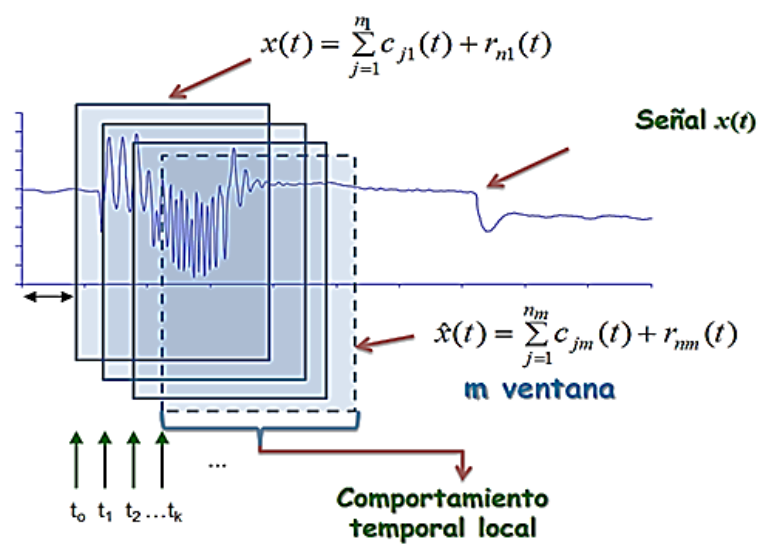

Figura 1 Ventana deslizante a lo largo de la señal medida Fuente: Elaboración propia

Esta técnica propuesta tiene la ventaja de contener poca carga computacional y asume que los data son estacionarios durante el análisis. Además, la elección apropiada de la longitud de la ventana es fundamental para los resultados obtenidos. Se prefiere una ventana de observación suficientemente corta con el fin de observar los cambios rápidos en las características temporales de la señal y simular un comportamiento estacionario de la señal sobre la duración de la ventana.

Con base a estas ideas, una EMD modificada es desarrollada y probada en varias señales reales. El método tiene la ventaja práctica que puede ser implementada tan pronto la señal ha sido recibida para el análisis de su comportamiento, obteniendo así una completa caracterización en línea (On-line).

Para evaluar los resultados obtenidos del método propuesto se calcula el error relativo rms para diferentes tamaños de ventanas, la cual está dada por:

$$
r m s=\sqrt{\frac{\int(x-\hat{x})^{2} d t}{\int x^{2} d t}}
$$

Donde $\hat{x}$ es una aproximación para la señal original $x$ y es obtenida por la suma de un número determinado de IMFs.

\section{HT vía filtro FIR}

El algoritmo de la aproximación a la transformada de Hilbert ideal a través de un filtro de respuesta-impulso-finito (FIR) se realiza mediante el uso de la función de ventana y se obtiene una aproximación al filtro FIR causal. El método de la ventana trunca la respuesta de impulso infinitamente largo multiplicándola por una secuencia finita llamada la función de ventana (Oppenheim \& Schafer, 1980).

$$
h[n]=h_{d}[n] w[n]=\left\{\begin{array}{cc}
h_{d}[n] & 0 \leq n \leq M \\
0 & \text { de otra forma }
\end{array}\right.
$$

En este caso, se usa la función de ventana de Kaiser para la longitud M del filtro (Kaiser, 1974):

$w[n]=\left\{\begin{array}{cc}\frac{I_{o}(\beta) \sqrt{\left.1-((n-M / 2) / M / 2)^{2}\right)}}{I_{o}(\beta)}, & 0 \leq n \leq M \\ 0, & \text { otra forma }\end{array}\right.$

Donde $I_{o}(\cdot)$ es la función de Bessel modificada de orden cero de primer tipo, la cual puede ser calculada a través de la expansión de la serie de potencia:

$$
I_{o}(x)=1+\sum_{k=1}^{\infty}\left[\frac{(x / 2)^{k}}{k !}\right]^{2}
$$

Los parámetros $M$ y $\beta$ del filtro en la ecuación (8) se determinan empíricamente y son las especificaciones para diseñar el filtro mediante el enfoque de la ventana de Kaiser.

Se crea la transformada del Hilbert $y_{j}[n]$, la cual está dada por la convolución (Oppenheim \& Schafer, 1980) entre la respuesta al impulso de un filtro FIR $h[n]$ y la $j t h \operatorname{IMF} c_{j}[n]$ :

$$
y_{j}[n]=\sum_{k=0}^{m-1} h[n] * c_{j}[n-k]
$$

Y se obtiene la $j t h$ señal analítica $z_{j}[n]$ cuya parte real es la $j t h$ IMF y la parte imaginaria es la función de cuadratura $y_{j}[n]$ :

$$
\begin{gathered}
z_{j}[n]=c_{j}[n]+i y_{j}[n]=A[n] e^{i \phi[n]} \\
c_{j}[n]=A[n] \cos (\phi[n]), \\
y_{j}[n]=A[n] \sin (\phi[n]) .
\end{gathered}
$$

Con esta metodología propuesta es posible conseguir la extracción de los patrones dinámicos a partir de mediciones PMU y lograr su completa caracterización en línea (On-line). 
En la Figura 2 se muestra una representación esquemática para la extracción de los atributos instantáneos.

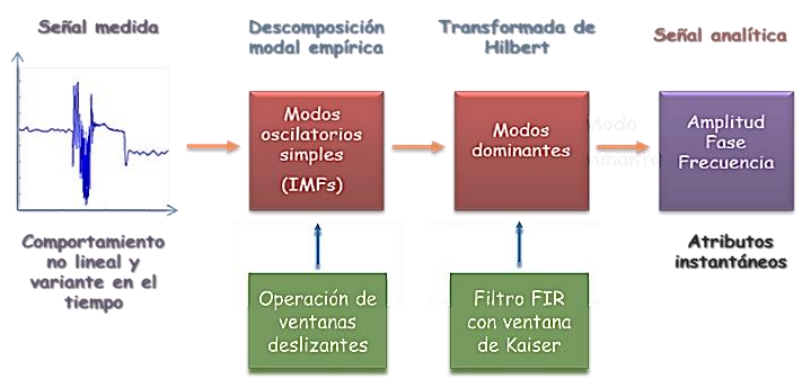

Figura 2 Procesamiento de una señal obtenida de PMU para determinar sus atributos instantáneos

Fuente: Elaboración propia

\section{Análisis de reducción de ruido en señales oscilatorias}

En las señales medidas siempre hay un nivel de ruido intrínseco. Por ello es importante disponer de ciertos criterios para la valorización del nivel de ruido que se hayan generado en los elementos del sistema eléctrico de potencia o introducidas por el equipo de medición, siendo en este caso las PMUs. La razón señal-ruido (SNR) permite comparar el nivel de señal y de ruido que existe al obtener información de una PMU.Se mide como la razón expresada en decibelios entre la potencia de la señal $P_{S}$ y la potencia del ruido $P_{n}$

$$
S N R=10 \log _{10}\left(\frac{P_{S}}{P_{n}}\right)
$$

En el dominio del tiempo, se tiene bien definido que las componentes IMFs más significativas son las que tienen mayor energía y las componentes que tienen mayor frecuencia, pero menor energía son ruido.

Partiendo de que la energía de la señal original es igual a la suma de las energías de las IMFs más la energía de su residuo, es necesario determinar el mínimo número de modos oscilatorios o IMFs que no contienen ruido.

Para lograr esto, se usa la energía local de cada IMF. La amplitud instantánea $A_{j}(t)$ y su correspondiente frecuencia instantánea $\omega_{j}(t)$ derivados de las IMFs a través de la transformada de Hilbert-Huang pueden proporcionar una completa distribución energíatiempo-frecuencia de los datos medidos. Así, la energía local del jth IMF está dada por (Huang, et al, 2003):

$$
E_{j}(t)=\frac{1}{2} A_{j}^{2}(t)
$$

La IMF con mayor energía será la IMF dominante y la IMF con menor energía es el residuo o la componente de ruido. Este criterio indica que podemos retener sólo los modos simples de oscilación sin ruido para analizar el conjunto de datos obtenidos de PMUs.

Después del proceso EMD, la señal original $x(t)$ es descompuesta en un finito número de IMFs y un residuo, entonces tenemos que, la energía de la señal bajo estudio $E_{x}(t)$ está dado por:

$$
E_{x}(t)=\sum_{j=1}^{n} E_{j}(t)+E_{r}(t)
$$

donde $E_{x}(t)$ es la energía total de las IMFs y el residuo, $E_{j}(t)$ es la energía del j-ésimo IMF y $E_{r}(t)$ es la energía del residuo. La señal reconstruida, no incluirá las IMFs con muy poca energía y alta frecuencia.

El error en la descomposición de la energía $\varepsilon$ está dado por:

$\varepsilon=\left|\frac{E_{j}-E_{x}}{E_{x}}\right|$

La variable $\varepsilon$ puede ser usada para indicar el índice de ortogonalidad de la EMD. Si $\varepsilon=0$ significa que las IMFs son completamente ortogonales. Y si $\varepsilon \rightarrow 0$ es que las IMFs son casiortogonales.

La técnica EMD demuestra la habilidad de actuar como un filtro para depurar las señales y mejore la calidad de la energía al eliminar el ruido.

\section{Resultados al aplicar a señales de PMUs}

Para evaluar el rendimiento y la capacidad del método propuesto se utilizó datos obtenidos de PMUs de un disturbio real en una subestación del sureste de México. La Figura 3 muestra la evolución temporal de la potencia activa en MW registrada en una interfaz crítica. Las mediciones se registraron durante 148 segundos recorridos a una velocidad de 6 muestras por segundo para un total de 888 muestras. 


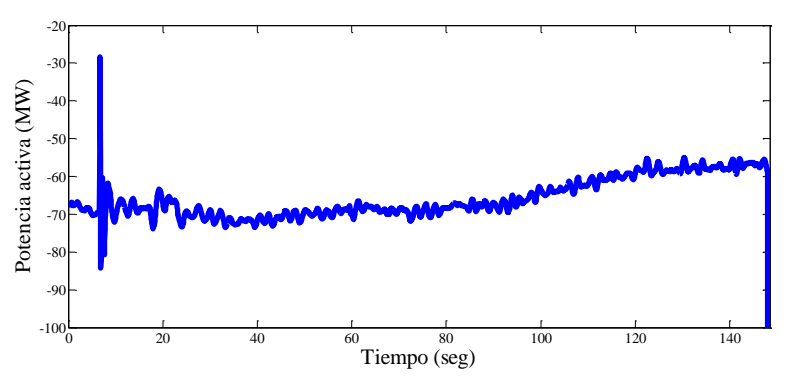

Figura 3 Comportamiento temporal de la potencia activa registrada durante la medición Fuente: Elaboración propia

Esta señal muestra una fuerte tendencia y exhibe no linealidades que complican el análisis. El espectro de Fourier para este evento se muestra en la Figura 4 en el cual se muestra el modo dominante en $0.48 \mathrm{~Hz}$. Se observa una pobre excitación de otros modos de diversas frecuencias en los datos que complican el análisis por ser ruido o pseudomodos, por lo que no serán examinados. Para comparar los resultados obtenidos con el enfoque propuesto, se hace uso de la técnica convencional HHT fuera de línea o post-mortem.

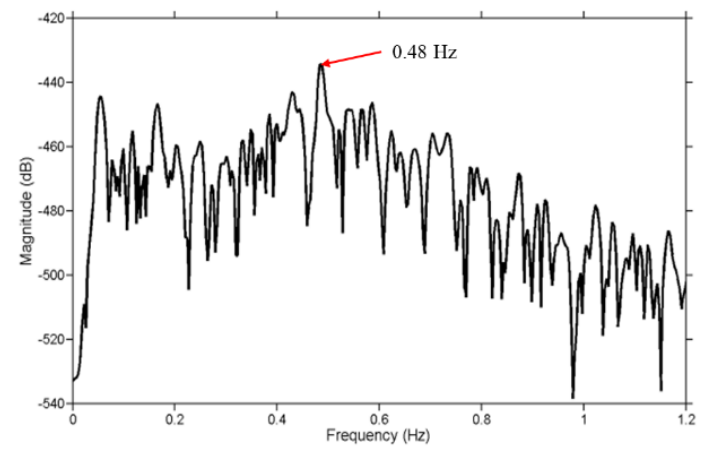

Figura 4 Espectro Discreto de Fourier obtenido de la medición

Fuente: Elaboración propia

En los resultados presentados, la EMD se calculó sobre una ventana de periodo 20 segundos, con un traslape del $25 \%$ de la longitud de la ventana y con parámetros $\beta=5.6533$, y $M=37$ obtenidos empíricamente.

La Figura 5 muestra el modo dominante IMF1 extraído con la EMD local. En el panel superior se caracteriza la amplitud instantánea de la señal usando ventana deslizante de 20 muestras. El panel inferior es un primer plano de la región transitoria (Detalle A), el cual es el período más violento del comportamiento del sistema y muestra la estimación de la transformada de Hilbert utilizando un filtro FIR de ventana de Kaiser.
La técnica propuesta es comparada con la estimación de la transformada de Hilbert utilizando el algoritmo de Park-McClellan tomando todos los datos, sin aplicar ventana deslizante (Parks \& McClellan, 1972). Se observa que la ventana de Kaiser proporciona una caracterización suave respecto al resultado obtenido con el filtro FIR de Park- McClellan.

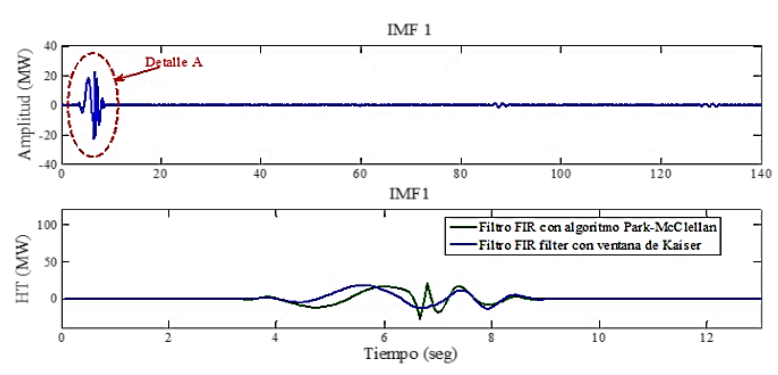

Figura 5 Arriba: amplitud instantánea de la primera IMF. Abajo: se amplía el detalle A y se compara la obtención de la transformada de Hilbert entre la técnica propuesta y el filtro FIR de Park-McClellan

Fuente: Elaboración propia

La fase y la frecuencia instantáneas de la IMF1 se muestran en las Figuras 6 y 7 respectivamente. El análisis en tiempo real de los datos medidos proporciona una mejor resolución en el dominio del tiempo que resulta en una caracterización más suave de los atributos y no contiene los errores numéricos que existen en la técnica convencional fuera de línea (Off-line) que emplea el algoritmo de Park-McClellan, el cual se manifiesta por picos falsos y largos.

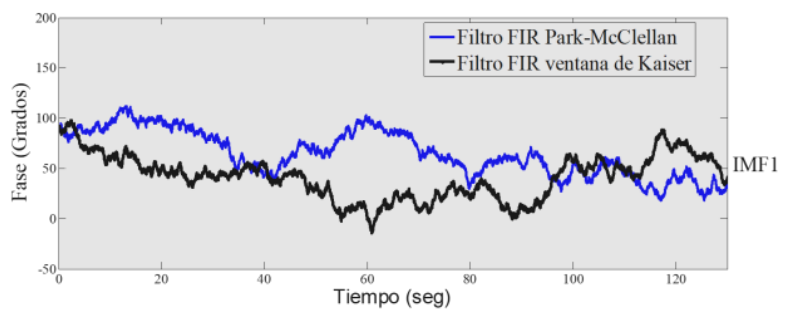

Figura 6 Comparación de la fase instantánea de IMF1 entre la propuesta del filtro de ventana de Kaiser y el filtro empleando el algoritmo Park-McClellan

Fuente: Elaboración propia

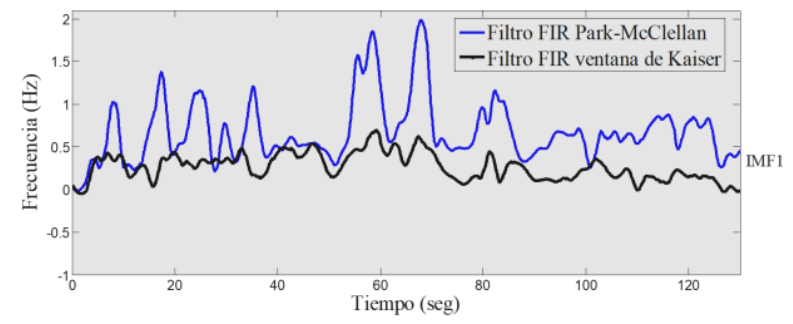

Figura 7 Comparación de la frecuencia instantánea de la IMF1 entre la propuesta del filtro de ventana de Kaiser y el filtro empleando el algoritmo Park-McClellan Fuente: Elaboración propia

LEZAMA-ZÁRRAGA, Francisco Román, CHAN GONZÁLEZ, Jorge de Jesús, SHIH, Meng Yen y CANTOCANUL, Roberto Carlos. Caracterización en línea de la dinámica temporal de señales para el monitoreo del sistema eléctrico de potencia. Revista de Energía Química y Física. 2020 
La señal original $x(t)$ fue reconstruida de acuerdo con la ecuación (1) tomando sólo las IMFs de interés más el residuo, es decir, tomando los términos $\sum_{j=1}^{p} c_{j}(t)+r_{n}(t)$ de la señal. Se evaluó el valor del error rms entre la señal original y señales reconstruidas obtenidas utilizando los cuatro primeros IMFs utilizando diferentes longitudes de la ventana deslizante sobrepuesta, con un traslape de $25 \%$ de los datos de cada ventana. Se observa en la Tabla 1 que el valor del error rms se reduce a medida que el tamaño de la ventana deslizante disminuye hasta llegar a 20 muestras y luego tiene un ligero incremento en la última ventana de prueba de 6 muestras.

\begin{tabular}{|l|l|l|}
\hline \multicolumn{1}{|c|}{ Método EMD } & \multicolumn{1}{|c|}{$\begin{array}{c}\text { Tamaño de la } \\
\text { ventana } \\
\text { deslizable } \\
\text { (muestras) }\end{array}$} & \multicolumn{1}{|c|}{$\begin{array}{c}\text { Error } \\
\text { (rms) }\end{array}$} \\
\hline $\begin{array}{l}\text { Enfoque } \\
\text { convencional } \\
\text { (fuera de línea) }\end{array}$ & $\begin{array}{l}\text { Todos los datos } \\
\text { medidos }\end{array}$ & 0.1644 \\
\hline $\begin{array}{l}\text { Enfoque propuesto } \\
\text { (en tiempo real) }\end{array}$ & 50 & 0.1282 \\
\cline { 2 - 3 } & 40 & 0.1215 \\
\cline { 2 - 3 } & 20 & 0.0543 \\
\hline
\end{tabular}

Tabla 1 Error rms en la aproximación Fuente: Elaboración propia

Debido a que el error rms fue menor para la ventana de 20 muestras, el estudio se realizó aplicando esta ventana deslizante.

\section{Resultados de reducción de ruido}

Para el análisis de reducción de ruido, la señal $x(t)$ obtenida de una PMU fue contaminada con ruido blanco gaussiano de $30 \mathrm{~dB}$ simulando un ruido ambiente común en los elementos del sistema eléctrico de potencia (ver parte inferior de la Figura 8). La técnica propuesta será aplicada y se analizarán los resultados para determinar si el método es preciso y capaz.

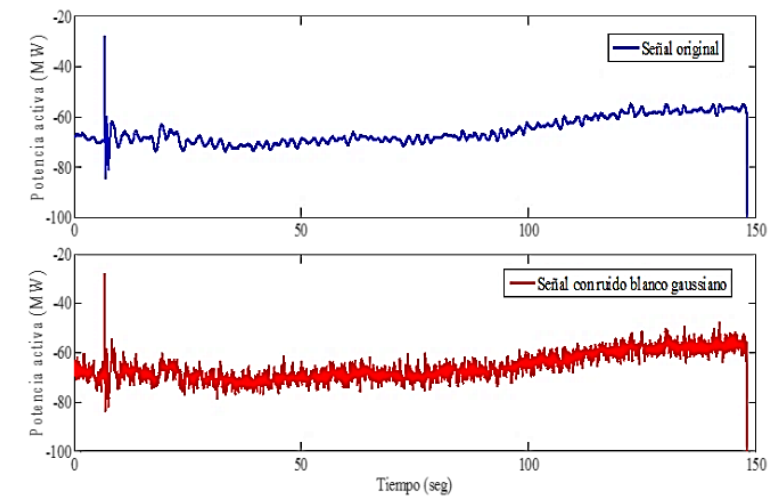

Figura 8 Señal de potencia afectada por ruido blanco gaussiano

Fuente: Elaboración propia

ISSN: 2410-3934

ECORFAN $®$ Todos los derechos reservados
La EMD local proporciona nueve IMFs y un residuo (ver Figura 9), los cuales están dados en orden descendente de nivel de frecuencia hasta que se llega a una oscilación monótona.

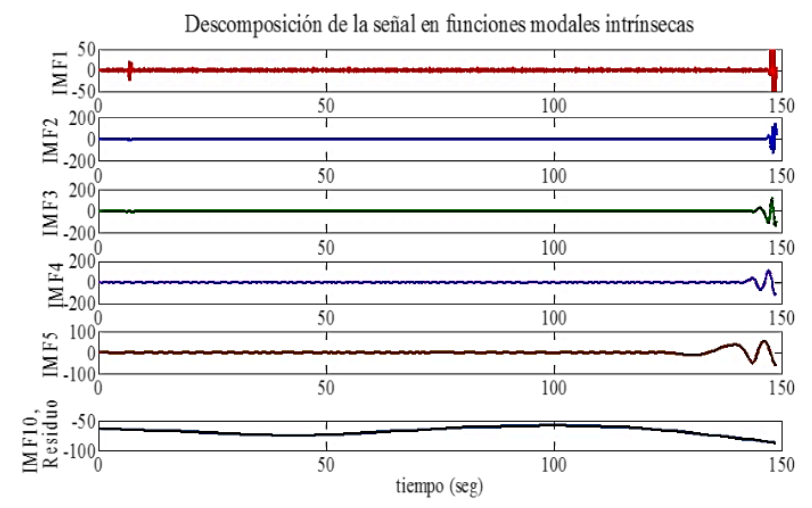

Figura 9 Señal de potencia descompuesta en IMFs y su residuo

Fuente: Elaboración propia

Durante el proceso de filtrado se aplica la ecuación (13) para determinar el nivel de energía de cada IMF. En la Tabla 2 se tiene los resultados. Se observa que la IMF1 es la que tiene la mayor frecuencia, pero su nivel de energía es pobre, esto se traduce que la IMF1 representa ruido y no se incluye en la reconstrucción de la señal.

\begin{tabular}{|c|c|c|c|c|}
\hline \multicolumn{5}{|c|}{ \% de Energía Local de cada IMF } \\
\hline IMF1 & IMF2 & IMF3 & IMF4 & IMF5 \\
\hline 0.0031 & 97.6214 & 0.9923 & 0.6354 & 0.2192 \\
\hline IMF6 & IMF7 & IMF8 & IMF9 & Residuo \\
\hline 0.1509 & 0.1398 & 0.0932 & 0.0843 & 0.0604 \\
\hline
\end{tabular}

Tabla 2 Contenido de energía en cada IMF y el residuo Fuente: Elaboración propia

Empleando la ecuación (12), se obtiene el nivel de ruido eliminado luego del proceso de filtrado siendo esta de $29.142 \mathrm{~dB}$ (ver figura inferior de Figura 10), por lo que sólo 0.858 dB se quedó en la señal reconstruida.

El valor del error rms de la señal reconstruida es de 0.0635 con respecto a la señal original y el error en la descomposición de la energía $\varepsilon$ es de 0.0237 corroborando que las IMFs son casi ortogonales.
LEZAMA-ZÁRRAGA, Francisco Román, CHANGONZÁLEZ, Jorge de Jesús, SHIH, Meng Yen y CANTOCANUL, Roberto Carlos. Caracterización en línea de la dinámica temporal de señales para el monitoreo del sistema eléctrico de potencia. Revista de Energía Química y Física. 2020 


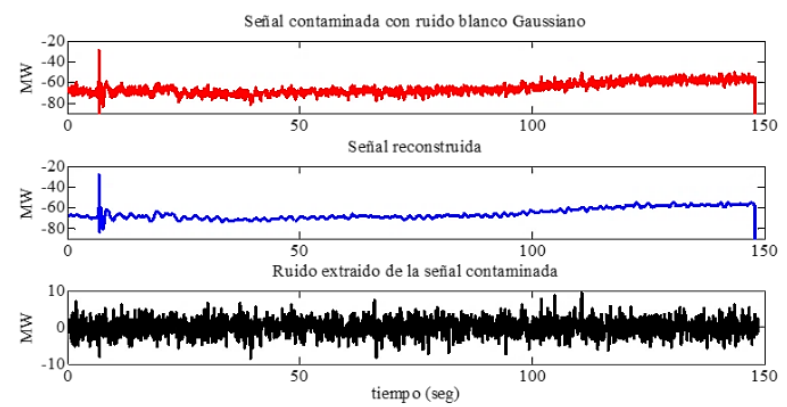

Figura 10 Señal de potencia que muestra el ruido extraído de la señal contaminada

Fuente: Elaboración propia

Los resultados sugieren que las implementaciones en línea (On-line) de la técnica propuesta son más precisas que los basados en la técnica convencional fuera de línea (Off-line) en la cual se toman todos los datos de la señal.

\section{Agradecimiento}

Los autores agradecen el apoyo y el esfuerzo de la Universidad Autónoma de Campeche para que sus investigadores difundan los proyectos de investigación en los cuales están inmersos.

\section{Conclusiones}

En este artículo se presenta un método eficaz en para el análisis de la dinámica local de oscilaciones transitorias utilizando una descomposición empírica modal (EMD) local y la transformada de Hilbert con un filtro FIR para ser implementado en línea (On-line), en la cual la señal es analizada por segmentos a través de ventanas deslizantes que se sobreponen unas sobre otras. Los resultados de la aplicación de la técnica demuestran que el método es preciso y capaz de identificar múltiples características modales con un profundo sentido físico. El enfoque propuesto es capaz de eliminar los efectos indeseables no lineales y, además, proporciona una excelente estimación de los atributos instantáneos y una aproximación importante a la señal original a través de la señal reconstruida.

Esta técnica de análisis de tiempofrecuencia-energía es muy precisa en el análisis de la dinámica local de oscilaciones transitorias y en la reducción de ruido en los sistemas eléctricos de potencia, la cual es posible implementar en análisis de oscilaciones en línea (On-line).
Se espera que el método pueda tener diversas aplicaciones en el área de análisis dinámico, incluyendo la determinación de la inestabilidad y la evaluación en línea del amortiguamiento.

\section{Referencias}

Aminifar, F., Fotuhi-Firuzabad, M., Safdarian, A., Davoudi, A. \& Shahidehpour, M. (2014). "Synchrophasor Measurement Technology in Power Systems: Panorama and State-of-theArt," in IEEE Access, vol. 2, pp. 1607-1628.

Byrne, R. H., Trudnowski, D. J., Neely, J. C., Elliott, R. T., Schoenwald, D. A. \& Donnelly, M. K. (2014). "Optimal locations for energy storage damping systems in the Western North American interconnect," 2014 IEEE PES General Meeting | Conference \& Exposition, National Harbor, MD, pp. 1-5.

De la O. Serna, J. A. (2007). "Dynamic phasor estimates for power system oscillations," IEEE Trans. Instrum. Meas., vol. 56, no. 5, pp. 16481657.

Girón-Lozano, L. V., de Jesús Salcedo-Hurtado, E. \& Pérez-Alcázar, G. A. (2020). “Análisis espectral del sismo del 27 de agosto de 2013 ocurrido en el Valle del Cauca, Colombia". Boletín de Geología, 42(2), pp. 49-67.

Hauer, F. \& DeSteesse, J. G. (2004). “A tutorial on detection and characterization of special behavior in large electric power systems," Pacific Northwest National Laboratory, Cambrige, MA, Rep. PNL-14655.

Huang, N. E., Shen, Z., Long, S. R., Wu, M. C., Shih, H. H., Zheng, Q., Yen, N. C., Tung, C. C. \& Liu, H. H. (1998). "The empirical mode decomposition and the Hilbert spectrum for nonlinear and non-stationary time series analysis," in Proc. R. Soc.Lon.A, vol. 454, pp. 903-995.

Huang, N. H., Wu, M. L., Long, S. R., Shen, S., Qu, W., Gloerson, P. \& Fan, K. (2003). "A confidence limit for the empirical mode decomposition and Hilbert spectral analysis," in Proc. R. Soc.Lon.A, vol. 31, pp. 417-457. 
Jiang, J. \& Zhang, Y. (2004). "A Novel Variable-Length Sliding Window Blockwise Least Squares Parameter Estimation Algorithm," in International Journal of Computers and Electrical Engineering, vol.30, No. 5, pp. 505-521.

Kaiser, J. F. \& Schafer, R. W. (1980). "On the use of the Io-sinh window for spectrum analysis," in IEEE Trans. Acoustics, Speech, and Signal Processing, Vol. 28, No. 1, pp. 105-107.

Messina, A. R. (Editor), (2009). "Inter-area Oscillations in Power Systems - A Nonlinear and No stationary Perspective," Ed. Springer Verlag. ISBN 978-0-387-89529-1, New York.

Messina, A. R., Vittal, V., Ruiz-Vega, D. \& Enriquez-Harper, G. (2006). "Interpretation and Visualization of Wide-Area PMU Measurements Using Hilbert Analysis," in IEEE Transactions on Power Systems, vol. 21, no. 4, pp. 1763-1771.

Oppenheim, A. V. \& Schafer, R. W. (1975). "Digital Signal Processing," Ed. Prentice Hall, ISBN 0-13-214635-5.

Parks, T. \& McClellan, J. (1972). "Chebyshev Approximation for Nonrecursive Digital Filters with Linear Phase," in IEEE Transactions on Circuit Theory, vol. 19, no. 2, pp. 189-194.

Phadke, A. G., Thorp, J. S. \& Adamiak, M. G. (1983). "A new measurement technique for tracking voltage phasors, local system frequency, and rate of change of frequency," IEEE Trans. Power App. Syst., vol. PAS-102, No. 5, pp. 1025-1038.

Pierre, J. W., Trudnowski, D. J., \& DeSteesse, J. G. (2007). "A perspective on WAMS analysis tools for tracking of oscillatory dynamics," 2007 IEEE Power Engineering Society General Meeting, Tampa, FL., pp. 1-10.

Rilling, G., Flandrin, P. \& Goncalves, P. (2003). "On empirical mode decomposition and its algorithm," in IEEE-EURASIP Workshop on Nonlinear Signal and Imege Processing NSIP03 .

Weideman, J. A. C. (1995). "Computing the Hilbert Transform On The Real Line," in Mathematics of Computation, Vol 64, No. 10, pp. 745-762.
LEZAMA-ZÁRRAGA, Francisco Román, CHANGONZÁLEZ, Jorge de Jesús, SHIH, Meng Yen y CANTOCANUL, Roberto Carlos. Caracterización en línea de la dinámica temporal de señales para el monitoreo del sistema eléctrico de potencia. Revista de Energía Química y Física. 2020 